\title{
Partner Selection Based on Optimal Power Allocation in Cooperative-Diversity Systems
}

\author{
Veluppillai Mahinthan, Member, IEEE, Lin Cai, Member, IEEE, \\ Jon W. Mark, Life Fellow, IEEE, and Xuemin (Sherman) Shen, Senior Member, IEEE
}

\begin{abstract}
We study how to appropriately match users for two-user cooperative-diversity (CD) systems deploying an optimal-power-allocation (OPA) strategy, considering both the amplify and forward and the regenerate and forward CD schemes. The OPA strategies, which can minimize the total energy consumption for the cooperating pair, are derived for both CD schemes. Then, we study the location of the optimal partner for a user and develop a novel nonbipartite stable matching algorithm with a performance that is very close to the optimal maximum weighted (MW) matching algorithm. The proposed matching algorithm is simple to implement, and its computational complexity is one order lower than that of the optimal MW matching algorithm. Numerical results show that with the OPA and the proposed matching algorithm, a 9 10 dB CD gain can be achieved, which is equivalent to prolonging the cell-phone-battery recharge time by about ten times.
\end{abstract}

Index Terms-Cooperative diversity (CD), matching algorithm, partner selection, power allocation, wireless networks.

\section{INTRODUCTION}

$\mathbf{F}$ UTURE wireless networks are expected to provide much higher data rates, energy efficiency, and reliability in a more cost-effective manner. With the state-of-the-art channel coding, energy and bandwidth efficiency in point-to-point communications can be made to approach the Shannon limit. On the other hand, battery life becomes the bottleneck for wireless devices. To meet the ever-increasing demand for higher data rates and longer battery life, a promising approach to further improve the energy and bandwidth efficiency is diversity reception. Cooperation among a group of users to transmit and relay the same signal can emulate a multiple transmit antenna environment to achieve spatial diversity gains. With the broadcast nature of the wireless channel, when a source transmits signals to a destination, neighboring users can also receive the signals. These neighboring users can relay the signals to the destination. The performance of cooperative diversity (CD) schemes heavily depends on the interuser channel condition. In this paper, we consider the cooperation between two users, i.e., two users relaying for each other, on the following bases: 1) the interuser channels

Manuscript received May 16, 2007. This work was supported in part by the Natural Sciences and Engineering Research Council (NSERC) of Canada under Grant RGPIN7779. The review of this paper was coordinated by Dr. Q. Zhang.

V. Mahinthan, J. W. Mark, and X. (S.) Shen are with the Centre for Wireless Communications, Department of Electrical and Computer Engineering, University of Waterloo, Waterloo, ON N2L 3G1, Canada (e-mail: mveluppi@ bbcr.uwaterloo.ca; jwmark@bbcr.uwaterloo.ca; xshen@bbcr.uwaterloo.ca).

L. Cai is with the Department of Electrical and Computer Engineering, University of Victoria, Victoria, BC V8W 2Y2, Canada (e-mail: cai@ece.uvic.ca).

Digital Object Identifier 10.1109/TVT.2007.905441 incur transmission errors in practice; 2) the implementation complexity increases with the number of users participating in the cooperation; and 3) the spectral efficiency of the wireless channel decreases with the number of participating users.

Although there has been a large body of work on CD reported in the literature in recent years, e.g., [1]-[13], the issue on how to optimize the system performance in a networked environment remains open. Optimizing the system performance involves the physical-layer CD scheme used (e.g., the amplify and forward CD schemes and the regenerate and forward CD schemes), the transmission power level of each user to satisfy their quality-of-service (QoS) requirements, and the partner selection or the matching strategy for the whole network. The physical-layer CD scheme used and the power level of each user should together decide the degree of CD energy gain of the cooperating pair of users or the energy gain of the individual user. The power needed for cooperative transmission or the power gain of cooperative transmission over noncooperative transmission can be used as weights for the matching (selection) algorithm at the network layer. In this paper, the terms partner selection and partner matching are interchangeably used.

Our objective is to minimize the overall energy-consumption rate in the network by appropriately grouping users and setting their power levels according to their QoS requirements. Since mobile users in wireless networks change their locations from time to time, by minimizing the energy spent by all users in the cell, the long term energy consumption rate of each user can be minimized so that the average battery recharge time can significantly be prolonged. In addition, the power allocation and matching algorithm should be with low overhead, low computational complexity, and easy implementation.

In this paper, we first formulate and solve the powerallocation problems of two-user cooperation in cellular networks, considering both the amplify and forward and the regenerate and forward $\mathrm{CD}$ systems. We also extend the optimal power allocation (OPA) problems with the constraint that the relaying power at the partner be equal to the source's transmission power. We refer to this as the equal power constraint, which is desired for static wireless networks like sensor networks, in which nodes are equipped with the same initial energy. The objective is to maximize the lifetime of the network; it is therefore necessary to minimize the energy consumption of the nodes with the maximum energy consumption rate. In this situation, equal power allocation (EPA) can maximize the lifetime of the pair. We then study the location of the optimal partner for a user and how to match the users to maximize the energy gain of the whole network. 


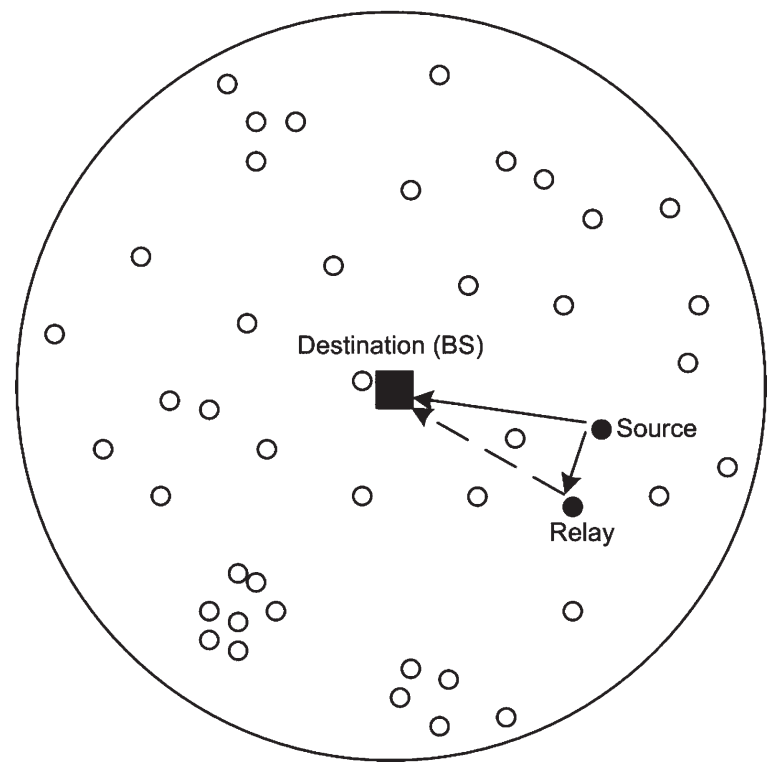

Fig. 1. Cellular network with user cooperation.

The main contributions of this paper are threefold. First, we formulate the optimization problem to maximize $\mathrm{CD}$ gain in a wireless network, taking into consideration the operational characteristics of different layers. Second, we obtain OPA solutions for general CD schemes to minimize the total energy consumption for a cooperating pair, with and without the EPA constraint, and then substantiate the analysis by calculating the optimal powers for the amplify and forward CD scheme proposed in [1] and the regenerate and forward CD scheme proposed in [2]. We further determine the locations of a user's best partner to minimize the energy consumption of the user and to maximize the $\mathrm{CD}$ energy gain of the pair. Third, the analytical results motivate us to propose a simple matching algorithm that can achieve near optimal performance. The power allocation strategies can directly be applied to various CD schemes proposed in the literature, and the proposed matching algorithm can easily be implemented in a centralized or distributed manner.

The remainder of this paper is organized as follows. Section II introduces the system model and the CD schemes considered. How to optimize the power allocation and the best partner's location is studied in Section III. Different matching algorithms are presented in Section IV. Numerical results are presented in Section V, followed by the related work and concluding remarks in Section VI and Section VII, respectively.

\section{SYSTEM MODEL}

Fig. 1 shows a wireless cellular network where the base station (BS) of a radio cell supports $N$ mobile users. A user is capable of cooperating with another user, i.e., cooperation between two active users. The BS and the mobile devices each has a single antenna. The uplink signals transmitted by the sender and relayed by the partner are combined at the BS using maximal ratio combining. The $\mathrm{CD}$ scheme thus emulates a "two-input one-output" situation.
The interuser and the user to BS channels are assumed to exhibit frequency nonselective Rayleigh fading and are independent of each other. They are also static over a frame interval and independently change from frame to frame, and each frame consists of $B$ bits. In addition, it is assumed that channel state information (CSI) is available at the respective receivers and that proper synchronization has been established.

There are two operational modes in performing cooperation between users to achieve CD: amplify and forward CD scheme and regenerate and forward $\mathrm{CD}$ scheme.

\section{A. Amplify and Forward CD Scheme}

With the amplify and forward CD scheme, the partner amplifies the signal received from the sender and retransmits it to the destination. The amplify and forward CD scheme proposed in [1] is used here. Binary-phase-shift-keying (BPSK) modulation is applied, and each receiver accumulates CSI and employs coherent detection. Each of the cooperating users is allocated with different frequency bands (centered at $f_{1}$ and $f_{2}$ ), and in each band, a user transmits signals in two time frames: One frame is dedicated for its own bits, and the other is for relaying the partner's bits. According to [1, eq. (13)], the approximate bit error probability (BEP) of user 1 for high signal-to-noise ratio (SNR) can be written as

$$
P_{\mathrm{b} 1}=\frac{3}{16 \bar{\gamma}_{1} \bar{\gamma}_{2}}+\frac{3}{16 \bar{\gamma}_{1} \bar{\gamma}_{1,2}} .
$$

The average received SNRs at the destination of the signals from users 1 and 2 are $\bar{\gamma}_{1}=\sigma_{1}^{2}\left(E_{\mathrm{b} 1}^{\mathrm{S}} / N_{0}\right)$ and $\bar{\gamma}_{2}=$ $\sigma_{2}^{2}\left(E_{\mathrm{b} 2}^{\mathrm{R}} / N_{0}\right)$, respectively, and the average received SNR at user 1 of the signal from user 2 is $\bar{\gamma}_{1,2}=\sigma_{1,2}^{2}\left(E_{\mathrm{b} 1}^{\mathrm{S}} / N_{0}\right)$. The $\sigma^{2}$ 's are the variances of the respective Rayleigh fading channel coefficients which are defined as the average CSI. $E_{\mathrm{b} 1}^{\mathrm{S}}$ and $E_{\mathrm{b} 2}^{\mathrm{R}}$ are the energies spent by the source (user 1) and the relay (user 2), respectively, in transmitting 1 bit for user 1 . The approximate BEP of user $2\left(P_{\mathrm{b} 2}\right)$ can similarly be expressed.

\section{B. Regenerate and Forward CD Scheme}

With the regenerate and forward CD scheme, the partner detects the received signal and transmits the regenerated version to the destination. We consider the quadrature signaling based adaptive regenerate and forward $\mathrm{CD}$ scheme [2] in which the partner's device employs cyclic redundancy check (CRC) to check the correctness of the received frame before making a decision of whether to forward the message. The asymptotic behavior of BEP of the CD scheme is derived in terms of the received SNR at the relay and at the destination and validated as a tight bound using simulation. According to [2, eq. (12)], the approximate BEP of user $1\left(P_{\mathrm{b} 1}\right)$, using $\mathrm{QPSK}^{1}$ modulation, is rewritten for high SNR as

$$
P_{\mathrm{b} 1}=\frac{K_{N}}{16 \bar{\gamma}_{1} \bar{\gamma}_{1,2}}+\frac{3}{16 \bar{\gamma}_{1} \bar{\gamma}_{2}}-\frac{3 K_{N}}{64 \bar{\gamma}_{1} \bar{\gamma}_{2} \bar{\gamma}_{1,2}}
$$

\footnotetext{
${ }^{1}$ Since the signal constellation is expanded for cooperation, it is equivalent to employing the BPSK by each user.
} 
where $K_{N}=\sum_{n=1}^{B}(1 / n)$ for a $B$-bit frame. The approximate BEP of user $2\left(P_{\mathrm{b} 2}\right)$ can similarly be expressed.

By using the quadrature-signaling-based regenerate and forward CD scheme [2], both the transmitting and the relaying bit energies at the respective user should be equal in order to avoid power imbalance in the in-phase and quadrature signaling. This is not the situation in other regenerate and forward CD schemes reported in the literature, e.g., [3], [7], [9], and [11].

\section{OPA AND PARTNER LOCATION}

Given the locations of the source and the relay, to satisfy the BEP requirements, the required power of the cooperating users depends on the quality of the interuser channel and the user-to-destination channels. How to minimize the total power consumption for the pair by appropriate power allocation is still an open issue. We first derive the OPA and EPA strategies for a general $\mathrm{CD}$ scheme and then substantiate the analysis by calculating the optimal powers for the amplify and forward CD scheme in [1] and the regenerate and forward CD scheme in [2].

\section{A. Power Allocation for Amplify and Forward CD Schemes}

Since power and energy are directly related, in what follows, we use the term power allocation even though the optimization problem is formulated as a minimization of the energy.

1) Optimal Power Allocation (OPA): Let $E_{\mathrm{b}_{i}}^{\mathrm{S}}$ and $E_{\mathrm{b}_{i}}^{\mathrm{R}}$ be the energies spent by user $i$ to transmit and relay a bit, respectively. For the amplify and forward CD scheme, the OPA problem is

$$
\begin{gathered}
\min \left(E_{\mathrm{b} 1}^{\mathrm{S}}+E_{\mathrm{b} 1}^{\mathrm{R}}+E_{\mathrm{b} 2}^{\mathrm{S}}+E_{\mathrm{b} 2}^{\mathrm{R}}\right) \\
\text { s.t. } P_{\mathrm{b} 1} \leq e_{1} \quad \text { and } \quad P_{\mathrm{b} 2} \leq e_{2} .
\end{gathered}
$$

Here, $e_{1}$ and $e_{2}$ are the maximum tolerable BEPs for users 1 and 2 , respectively.

$P_{\mathrm{b} 1}$ is a function of $E_{\mathrm{b} 1}^{\mathrm{S}}$ and $E_{\mathrm{b} 2}^{\mathrm{R}}$, whereas $P_{\mathrm{b} 2}$ is a function of $E_{\mathrm{b} 2}^{\mathrm{S}}$ and $E_{\mathrm{b} 1}^{\mathrm{R}}$. Since each user can use different power levels for transmitting and relaying, $E_{\mathrm{b}}^{\mathrm{S}}$ and $E_{\mathrm{b}}^{\mathrm{R}}$ are independent of each other. Therefore, the optimization problem can be decomposed into two independent optimization problems as follows:

$$
\begin{aligned}
& \min \left(E_{\mathrm{b} 1}^{\mathrm{S}}+E_{\mathrm{b} 2}^{\mathrm{R}}\right) \\
& \text { s.t. } P_{\mathrm{b} 1}\left(E_{\mathrm{b} 1}^{\mathrm{S}}, E_{\mathrm{b} 2}^{\mathrm{R}}\right) \leq e_{1}
\end{aligned}
$$

and

$$
\begin{aligned}
& \min \left(E_{\mathrm{b} 2}^{\mathrm{S}}+E_{\mathrm{b} 1}^{\mathrm{R}}\right) \\
& \text { s.t. } P_{\mathrm{b} 2}\left(E_{\mathrm{b} 2}^{\mathrm{S}}, E_{\mathrm{b} 1}^{\mathrm{R}}\right) \leq e_{2} .
\end{aligned}
$$

Note that for a given BEP of a user, the transmission power of the user is always a nonincreasing continuous convex function of the relay power of its partner and vice versa, as shown in Fig. 2. To minimize the energy consumption $\left(E_{\mathrm{b} 1}^{\mathrm{S}}+E_{\mathrm{b} 2}^{\mathrm{R}}\right)$ with constraint $P_{\mathrm{b} 1} \leq e_{1}$, the optimal operating point is the tangent

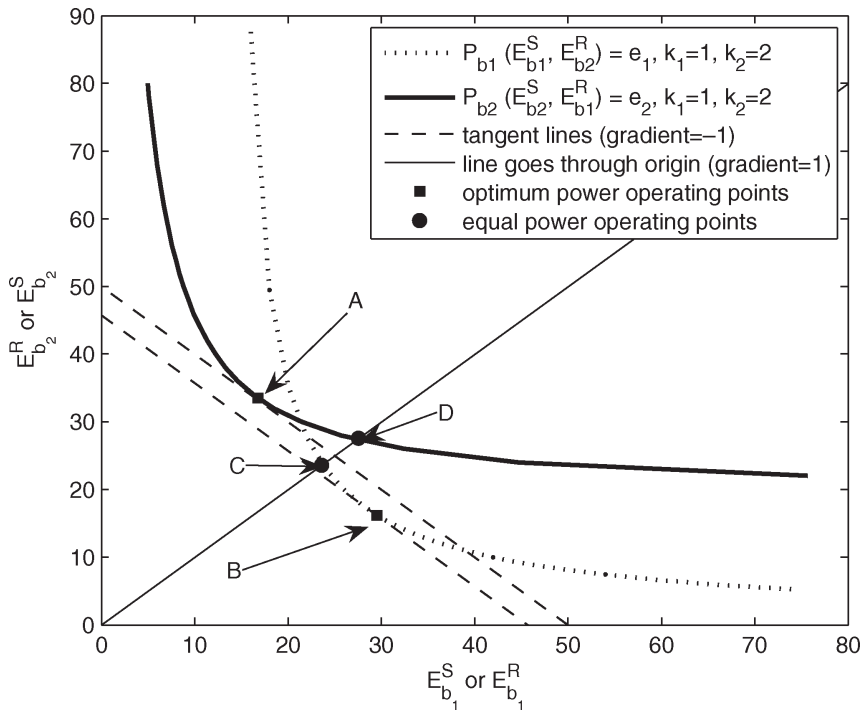

Fig. 2. Power allocation problem of the amplify and forward CD scheme.

point of the dotted curve touched by the line with slope -1 , i.e., point $B$ in Fig. 2. This is because all the points on the line with slope -1 correspond to the same value of $\left(E_{\mathrm{b} 1}^{\mathrm{S}}+E_{\mathrm{b} 2}^{\mathrm{R}}\right)$, and all other points on the dotted curve have higher total energy level than that of point $B$. Similarly, the solution for problem (5) is the tangent point of the solid curve touched by the line with slope -1, i.e., point $A$ in Fig. 2.

We verify this by using the amplify and forward CD scheme proposed in [1]. Let $k_{1}=\sigma_{1,2}^{2} / \sigma_{1}^{2}$ and $k_{2}=\sigma_{2,1}^{2} / \sigma_{2}^{2}$. Because of the broadcast nature of the channel, $\bar{\gamma}_{1,2}=k_{1} \bar{\gamma}_{1}$, and $\bar{\gamma}_{2,1}=k_{2} \bar{\gamma}_{2}$. Furthermore, $\sigma_{2}^{2}=(k 1 / k 2) \sigma_{1}^{2}$ because of the reciprocity of the interuser channel $\left(\sigma_{1,2}^{2}=\sigma_{2,1}^{2}\right)$. The minimal relay power can be obtained as a function of the transmission power and the required BEP:

$$
E_{\mathrm{b} 2}^{\mathrm{R}}=\frac{3 k_{2} N_{0}^{2} E_{\mathrm{b} 1}^{\mathrm{S}}}{\left(16 k_{1} P_{\mathrm{b} 1} \sigma_{1}^{4}\left(E_{\mathrm{b} 1}^{\mathrm{S}}\right)^{2}-3 N_{0}^{2}\right)} .
$$

By solving $d E_{\mathrm{b} 2}^{\mathrm{R}} / d E_{\mathrm{b} 1}^{\mathrm{S}}=-1$, the optimal solutions of $E_{\mathrm{b} 1}^{\mathrm{S}}$ and $E_{\mathrm{b} 2}^{\mathrm{R}}$ can be written as

$$
\begin{aligned}
E_{\mathrm{b} 1}^{\mathrm{S}}= & \frac{N_{0}}{\sigma_{1}^{2}}\left(\frac{6+3 k_{2}+\sqrt{9 k_{2}^{2}+72 k_{2}}}{32 k_{1} P_{\mathrm{b} 1}}\right)^{1 / 2} \\
E_{\mathrm{b} 2}^{\mathrm{R}}= & \left(\frac{2 k_{2}}{k_{2}+\sqrt{k_{2}^{2}+8 k_{2}}}\right) \frac{N_{0}}{\sigma_{1}^{2}} \\
& \times\left(\frac{6+3 k_{2}+\sqrt{9 k_{2}^{2}+72 k_{2}}}{32 k_{1} P_{\mathrm{b} 1}}\right)^{1 / 2} .
\end{aligned}
$$

Similarly, for user 2

$$
E_{\mathrm{b} 1}^{\mathrm{R}}=\frac{3 k_{1} k_{2} N_{0}^{2} E_{\mathrm{b} 2}^{\mathrm{S}}}{\left(16 k_{1}^{2} P_{\mathrm{b} 2} \sigma_{1}^{4}\left(E_{\mathrm{b} 2}^{\mathrm{S}}\right)^{2}-3 k_{2} N_{0}^{2}\right)} .
$$


By solving $d E_{\mathrm{b} 1}^{\mathrm{R}} / d E_{\mathrm{b} 2}^{\mathrm{S}}=-1$, the optimal solutions of $E_{\mathrm{b} 2}^{\mathrm{S}}$ and $E_{\mathrm{b} 1}^{\mathrm{R}}$ can be written as

$$
\begin{aligned}
E_{\mathrm{b} 2}^{\mathrm{S}}= & \frac{N_{0}}{\sigma_{1}^{2}}\left(\frac{6 k_{2}+3 k_{1} k_{2}+\sqrt{9 k_{2}^{2} k_{1}^{2}+72 k_{2}^{2} k_{1}}}{32 k_{1}^{2} P_{\mathrm{b} 1}}\right)^{1 / 2} \\
E_{\mathrm{b} 1}^{\mathrm{R}}= & \left(\frac{2 k_{1}}{k_{1}+\sqrt{k_{1}^{2}+8 k_{1}}}\right) \frac{N_{0}}{\sigma_{1}^{2}} \\
& \times\left(\frac{6 k_{2}+3 k_{1} k_{2}+\sqrt{9 k_{2}^{2} k_{1}^{2}+72 k_{2}^{2} k_{1}}}{32 k_{1}^{2} P_{\mathrm{b} 1}}\right)^{1 / 2} .
\end{aligned}
$$

2) Equal Power Allocation: To maximize the lifetime of the cooperative pair in static networks, the EPA is preferred. Since the energy usage level of both users is the same, we should add another constraint to the OPA. The optimization problem with equal power constraints can be formulated as

$$
\begin{aligned}
& \min \left(E_{\mathrm{b} 1}^{\mathrm{S}}+E_{\mathrm{b} 2}^{\mathrm{R}}\right) \\
& \text { s.t. } P_{\mathrm{b} 1}\left(E_{\mathrm{b} 1}^{\mathrm{S}}, E_{\mathrm{b} 2}^{\mathrm{R}}\right) \leq e_{1} \text { and } E_{\mathrm{b} 1}^{\mathrm{S}}-E_{\mathrm{b} 2}^{\mathrm{R}}=0
\end{aligned}
$$

and

$$
\begin{aligned}
& \min \left(E_{\mathrm{b} 2}^{\mathrm{S}}+E_{\mathrm{b} 1}^{\mathrm{R}}\right) \\
& \text { s.t. } P_{\mathrm{b} 2}\left(E_{\mathrm{b} 2}^{\mathrm{S}}, E_{\mathrm{b} 1}^{\mathrm{R}}\right) \leq e_{2} \text { and } E_{\mathrm{b} 2}^{\mathrm{S}}-E_{\mathrm{b} 1}^{\mathrm{R}}=0 .
\end{aligned}
$$

Obviously, for (14), the optimal operating point is the intersection of the equal power line (the line with slope 1 and passing through the origin) and the dotted curve, i.e., point $C$ in Fig. 2. For the CD scheme in [1], the optimal solution is given by

$$
E_{\mathrm{b} 1}^{\mathrm{S}}=E_{\mathrm{b} 2}^{\mathrm{R}}=\sqrt{\frac{3 N_{0}^{2}\left(1+k_{2}\right)}{16 k_{1} P_{\mathrm{b} 1} \sigma_{1}^{4}}} .
$$

For (15), the intersection point $D$ in Fig. 2 corresponds to the optimal solution given by

$$
E_{\mathrm{b} 2}^{\mathrm{S}}=E_{\mathrm{b} 1}^{\mathrm{R}}=\sqrt{\frac{3 N_{0}^{2} k_{2}\left(1+k_{1}\right)}{16 k_{1}^{2} P_{\mathrm{b} 2} \sigma_{1}^{4}}} .
$$

\section{B. Power Allocation for Regenerate and Forward CD Scheme}

1) Optimal Power Allocation: For the regenerate and forward CD schemes, if the user can have different power levels for transmitting and relaying [3], [7], [9], [11], the optimization problem can be solved using the same approach as in the previous section. In a practical situation, when a user must have the same power level for transmitting and relaying due to implementation concern [2], the power allocation problem can be formulated as the following convex optimization problem:

$$
\begin{aligned}
\min & \left(E_{\mathrm{b} 1}+E_{\mathrm{b} 2}\right) \\
\text { s.t. } & P_{\mathrm{b} 1}\left(E_{\mathrm{b} 1}, E_{\mathrm{b} 2}\right) \leq e_{1} \text { and } P_{\mathrm{b} 2}\left(E_{\mathrm{b} 1}, E_{\mathrm{b} 2}\right) \leq e_{2}
\end{aligned}
$$

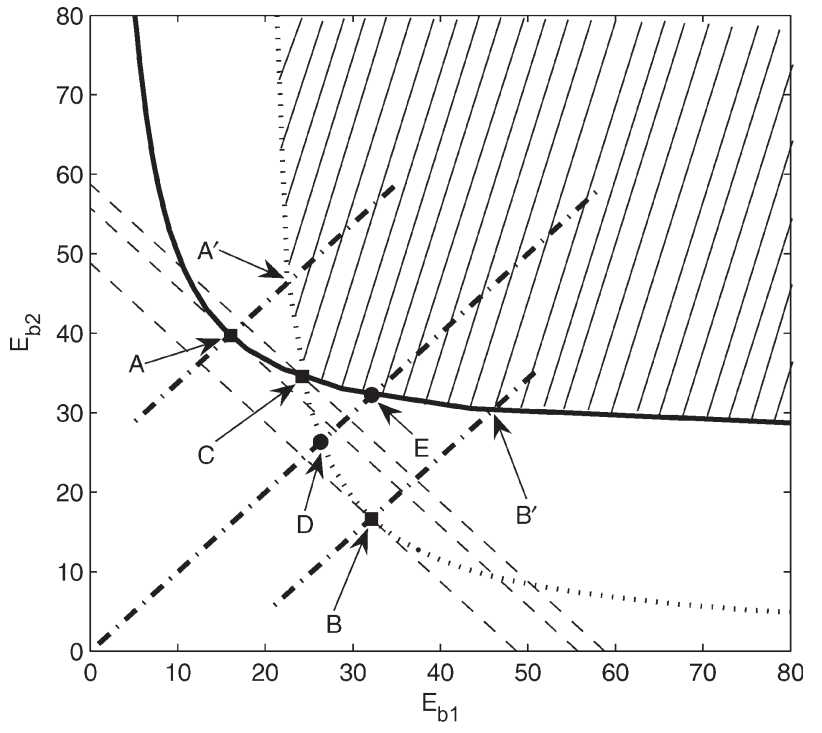

Fig. 3. Power allocation problem of the regenerate and forward CD scheme.

where $e_{1}$ and $e_{2}$ are the maximum tolerable BEPs for users 1 and 2, respectively, $E_{\mathrm{b} 1}^{\mathrm{S}}=E_{\mathrm{b} 2}^{\mathrm{R}}=E_{\mathrm{b} 1}$, and $E_{\mathrm{b} 2}^{\mathrm{S}}=E_{\mathrm{b} 1}^{\mathrm{R}}=E_{\mathrm{b} 2}$. The optimization problem can be solved as follows. As mentioned earlier, the transmission power of the user is always a nonincreasing continuous convex function of the relay power of its partner. For a given BEP, if $E_{\mathrm{b} 1}$ is smaller, $E_{\mathrm{b} 2}$ must be larger and vice versa. As shown in Fig. 3, to guarantee that $P_{\mathrm{b} 1} \leq e_{1}$ and $P_{\mathrm{b} 2} \leq e_{2}$, the energy levels of the two users should be in the intersection of the region above ${ }^{2}$ the dotted and the solid curves, which is shown as the shaded area. To select the optimum operating point that minimizes the energy consumption $\left(E_{\mathrm{b} 1}+E_{\mathrm{b} 2}\right)$ with the constraints $P_{\mathrm{b} 1} \leq e_{1}$ and $P_{\mathrm{b} 2} \leq e_{2}$, we should consider the following three cases.

Case I) The optimal operating point that satisfies $P_{\mathrm{b} 1}=e_{1}$ only is the tangent point of the dotted curve touched by the line with slope -1 , i.e., point $B$ in Fig. 3. This is because all the points on the line with (dashed lines) slope -1 correspond to the same value of $\left(E_{\mathrm{b} 1}+E_{\mathrm{b} 2}\right)$, and all other points on the dotted curve have higher total energy levels than that of point $B$. If point $B$ also satisfies $P_{\mathrm{b} 2} \leq e_{2}$, point $B$ is the optimal solution for the OPA problem.

Case II) If the condition in case I) is not satisfied, we can identify the optimal operating point to minimize the total energy with the constraint $P_{\mathrm{b} 2}=e_{2}$ only, which is the tangent point of the solid curve touched by the line with slope -1 , i.e., point $A$ in Fig. 3. If point $A$ also satisfies $P_{\mathrm{b} 1} \leq e_{1}$, point $A$ is the optimal solution for the OPA problem.

Case III) If the conditions in cases I) and II) are not satisfied, according to the following theorem, the two curves must intersect at a point which is the optimal operating point of the OPA problem.

\footnotetext{
${ }^{2}$ When a point is "above" another point, we mean that the total energy consumption of the two users corresponding to the former one is higher.
} 
Theorem 1: Let point $A\left(E_{\mathrm{b} 1}^{A}, E_{\mathrm{b} 2}^{A}\right)$ be the tangent point of (the solid curve in Fig. 3) $P_{\mathrm{b} 2}\left(E_{\mathrm{b} 1}, E_{\mathrm{b} 2}\right)=e_{2}$ touched by the line with slope -1 , and let $B\left(E_{\mathrm{b} 1}^{B}, E_{\mathrm{b} 2}^{B}\right)$ be the tangent point of (the dotted curve in Fig. 3) $P_{\mathrm{b} 1}\left(E_{\mathrm{b} 1}, E_{\mathrm{b} 2}\right)=e_{1}$ touched by the line with slope -1 . If $P_{\mathrm{b} 1}\left(E_{\mathrm{b} 1}^{A}, E_{\mathrm{b} 2}^{A}\right)>e_{1}$ and $P_{\mathrm{b} 2}\left(E_{\mathrm{b} 1}^{B}, E_{\mathrm{b} 2}^{B}\right)>e_{2}$, the two functions $P_{\mathrm{b} 1}\left(E_{\mathrm{b} 1}, E_{\mathrm{b} 2}\right)=e_{1}$ and $P_{\mathrm{b} 2}\left(E_{\mathrm{b} 1}, E_{\mathrm{b} 2}\right)=e_{2}$ must intersect at a point labeled $C$. $C$ is located between the two parallel lines with slope 1: one passing through point $A$ and the other passing through point $B$. Moreover, $C$ is the optimal solution of the OPA problem in (18).

Proof: The line passing through point $A$ with slope 1 intersects the solid curve at point $A^{\prime}$ which must be above point $A$; otherwise, point $A$ will satisfy the condition $P_{\mathrm{b} 1} \leq e_{1}$ (case I), which contradicts the condition of the theorem. The line passing through point $B$ with slope 1 intersects the solid curve at point $B^{\prime}$ which must be above point $B$; otherwise, point $B$ will satisfy the condition $P_{\mathrm{b} 2} \leq e_{2}$ (case II), which contradicts the condition of the theorem. Given that $P_{\mathrm{b} 1}\left(E_{\mathrm{b} 1}, E_{\mathrm{b} 2}\right)=e_{1}$ and $P_{\mathrm{b} 2}\left(E_{\mathrm{b} 1}, E_{\mathrm{b} 2}\right)=e_{2}$ are continuous convex functions, $A B^{\prime}$ and $B A^{\prime}$ must intersect at one and only one point, i.e., point $C$. On the solid curve, from point $A$ to $B^{\prime}, E_{\mathrm{b} 1}+E_{\mathrm{b} 2}$ monotonically increases, and any point between $A$ and $C$ cannot satisfy $P_{\mathrm{b} 1}\left(E_{\mathrm{b} 1}, E_{\mathrm{b} 2}\right) \leq e_{1}$. Thus, $C$ is the point with the minimum $E_{\mathrm{b} 1}+E_{\mathrm{b} 2}$ and satisfies both constraints, and it is the optimum solution.

By using the CD scheme proposed in [2], (2) can be written as

$$
E_{\mathrm{b} 2}=\frac{\left(12 k_{1} k_{2} \sigma_{1}^{2} N_{0}^{2} E_{\mathrm{b} 1}-3 K_{N} k_{2} N_{0}^{3}\right)}{\left(64 k_{1}^{2} P_{\mathrm{b} 1} \sigma_{1}^{6} E_{\mathrm{b} 1}^{2}-8 K_{N} k_{1} N_{0}^{2} \sigma_{1}^{2}\right)}
$$

The coordinates of point $B$ can be obtained by solving $d E_{\mathrm{b} 2} / d E_{\mathrm{b} 1}=-1$.

Similarly, for user 2

$$
E_{\mathrm{b} 1}=\frac{\left(12 k_{1} k_{2} \sigma_{1}^{2} N_{0}^{2} E_{\mathrm{b} 2}-3 K_{N} k_{2} N_{0}^{3}\right)}{\left(64 k_{1}^{2} \sigma_{1}^{6} P_{\mathrm{b} 2} E_{\mathrm{b} 2}^{2}-8 K_{N} k_{2} N_{0}^{2} \sigma_{1}^{2}\right)} .
$$

The coordinates of point $A$ can be obtained by solving $d E_{\mathrm{b} 1} / d E_{\mathrm{b} 2}=-1$.

The coordinates of the intersection point of $P_{\mathrm{b} 1}=e_{1}$ and $P_{\mathrm{b} 2}=e_{2}$, which is point $C$, can be obtained from the solutions of a fifth order polynomial, which is obtained by substituting $E_{\mathrm{b} 2}$ in (19) by the right-hand side of (20). The coordinates of points $A, B$, and $C$ can numerically be solved.

2) Equal Power Allocation: For the case of EPA, both users are transmitting at equal power level. This requires one more constraint to the previously given OPA, and the optimization problem can be formulated as

$$
\begin{aligned}
& \min \left(E_{\mathrm{b} 1}+E_{\mathrm{b} 2}\right) \\
& \text { s.t. } P_{\mathrm{b} 1}\left(E_{\mathrm{b} 1}, E_{\mathrm{b} 2}\right) \leq e_{1}, P_{\mathrm{b} 2}\left(E_{\mathrm{b} 1}, E_{\mathrm{b} 2}\right) \leq e_{2}, \\
& \quad \text { and } E_{\mathrm{b} 1}-E_{\mathrm{b} 2}=0 .
\end{aligned}
$$

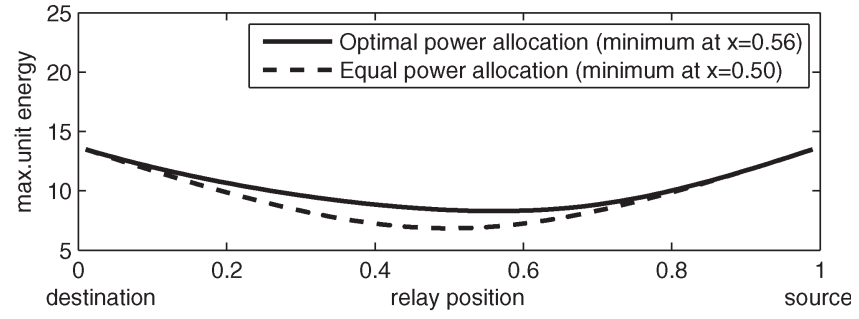

(a)

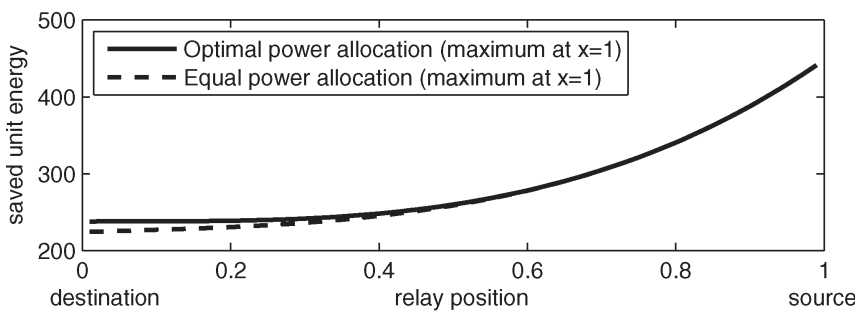

(b)

Fig. 4. One-dimensional analysis of the amplify and forward CD scheme.

As shown in Fig. 3, the shaded area can guarantee the BEPs of the pair of users, and the intersection point of the equal power line with the boundary of the shaded area, which is point $E$, corresponds to the optimal solution.

Using the regenerate and forward CD scheme in [2], by substituting $E_{\mathrm{b} 1}=E_{\mathrm{b} 2}$ into (19) and (20), the coordinates of points $D$ and $E$ can be obtained, respectively. Between $D$ and $E$, the point with the higher power level can satisfy both conditions $P_{\mathrm{b} 1} \leq e_{1}$ and $P_{\mathrm{b} 2} \leq e_{2}$, and it will be the optimal equal power solution for the regenerate and forward $\mathrm{CD}$ scheme in [2].

\section{Optimal Partner Location}

To facilitate the derivation of the matching algorithm, we study the location of the optimal partner based on the OPA. It is trivial to prove that the optimal partners' locations, which maximize the cooperative energy gain of the pair and minimize the power consumption rate of the user, are both on the line connecting the source and the destination (BS). Therefore, in the following, we determine the optimal locations of the partners in the one-dimensional line segment between the source and the destination.

Assume that the average CSI is proportional to the respective distance raised to the power $\alpha$, i.e., $\sigma_{1}^{2} \propto d_{1}^{-\alpha}, \sigma_{2}^{2} \propto d_{2}^{-\alpha}$, and $\sigma_{1,2}^{2} \propto d_{1,2}^{-\alpha}$, where the path loss exponent $\alpha$ takes the value between two and six. Define maximum unit energy $=$ $\max \left\{E_{\mathrm{b} 1}^{\mathrm{S}}, E_{\mathrm{b} 2}^{\mathrm{S}}, E_{\mathrm{b} 1}^{\mathrm{R}}, E_{\mathrm{b} 2}^{\mathrm{R}}\right\}$, and saved unit energy $=E_{\mathrm{b} 1}^{\mathrm{no}}+$ $E_{\mathrm{b} 2}^{\mathrm{no}}-E_{\mathrm{b} 1}^{\mathrm{S}}-E_{\mathrm{b} 2}^{\mathrm{S}} E_{\mathrm{b} 1}^{\mathrm{R}}-E_{\mathrm{b} 2}^{\mathrm{R}}$. Consider a pair of users; user 2 is located on the line between the BS and the user 1 . By substituting $P_{\mathrm{b} 1}=P_{\mathrm{b} 2}=10^{-3}, B=128, N_{0}=1$ unit power $/ \mathrm{Hz}$, the CSIs in terms of normalized distance, and $\alpha=3$, we demonstrate the trends of the maximum and the saved unit energies for the CD schemes with OPA and EPA, respectively.

In Figs. 4 and 5, the $x$-axis represents the location of the partner while the destination is located at $x=0$, and the source is 


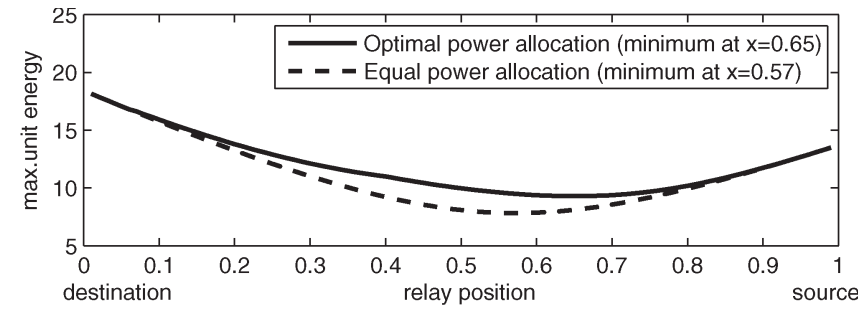

(a)

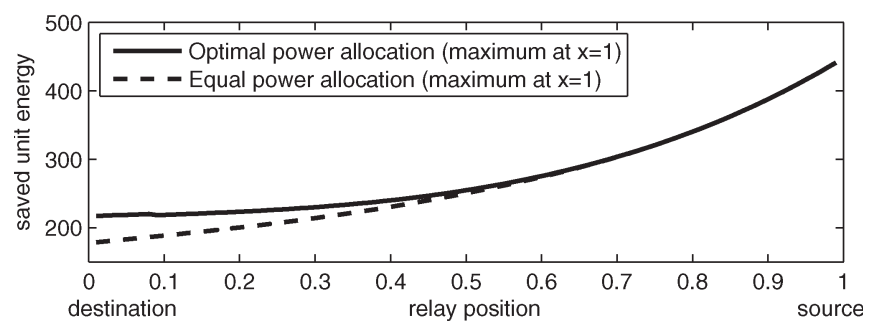

(b)

Fig. 5. One-dimensional analysis of the regenerate and forward CD scheme.

located at $x=1$ (the source-destination distance is normalized to 1 unit). The $y$-axis of Fig. 4(a) represents the maximum unit energy of the pair with the amplify and forward CD scheme, and the optimal locations of the partner for minimizing the energy consumption of the source are $x=0.58$ and $x=0.50$ for the OPA and the EPA, respectively. Fig. 4(b) shows that the saved unit energy of the cooperative pair for both powerallocation schemes is maximized when $x=1$. From a user's point of view, its optimal partner's location is close to the midpoint between itself and the destination; on the other hand, to maximize the total energy saving for the pair of users, the two users should be collocated. As shown in Fig. 5(a), for the case of the regenerate and forward CD scheme, the maximum unit energy is minimized when $x=0.65$ and $x=0.57$ for the OPA and the EPA, respectively, and the behavior of the saved unit energy in Fig. 5(b) is similar to that of the amplify-andforward-based CD scheme.

A two-dimensional plot of cooperative region of both the regenerate and forward and the amplify and forward schemes, considering the source's gain, is shown in Figs. 6 and 7, respectively. The solid curves in the figures correspond to the case with OPA, and the dotted curves correspond to the case with EPA. It can be seen that the OPA enlarges the cooperativeregion contour with a fixed $\mathrm{CD}$ gain for the pair, particularly when the partner is far away from the source, and that their optimal power levels are quite different. The contours of the cooperative region in these figures can help locate the best partner of any user.

\section{Matching Algorithms}

Choosing pairs of cooperating users is known as matching problems on graphs [9], [10], [14]. Let $\mathcal{G}=\{\mathcal{V}, \mathcal{E}\}$ be a graph, where $\mathcal{V}$ is a set of vertexes, and $\mathcal{E} \subseteq \mathcal{V} \times \mathcal{V}$ is a set of edges between vertexes. Each mobile user in a cell is represented as a vertex. The $(i, j)$ th edge $e_{i, j} \in \mathcal{E}$ has a weight $w\left(e_{i, j}\right)$, which depends on the matching algorithm. A subset $\mathcal{S}$ of $\mathcal{E}$ is called

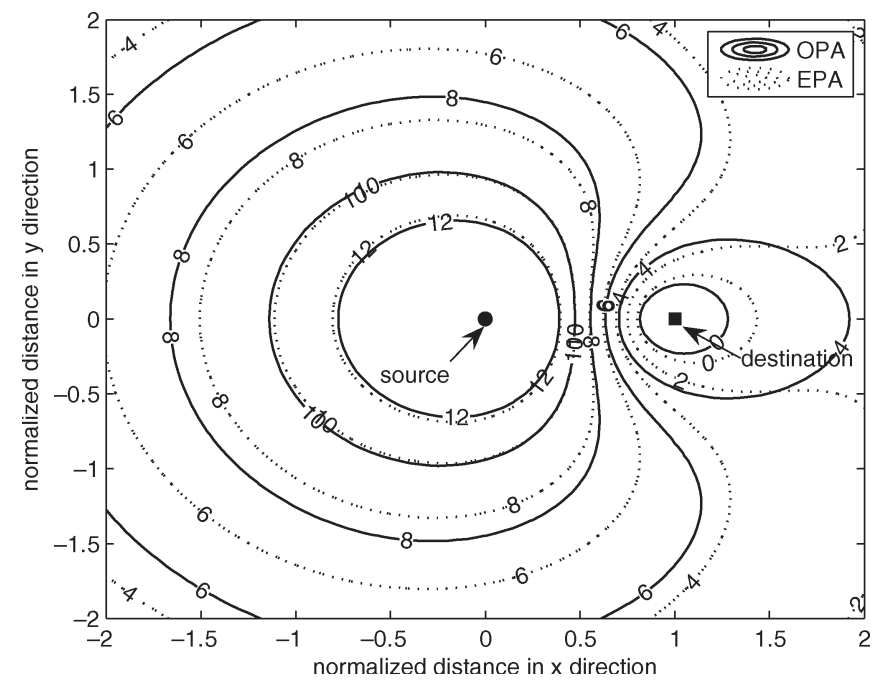

Fig. 6. Cooperative region of the amplify and forward scheme for both power allocation.

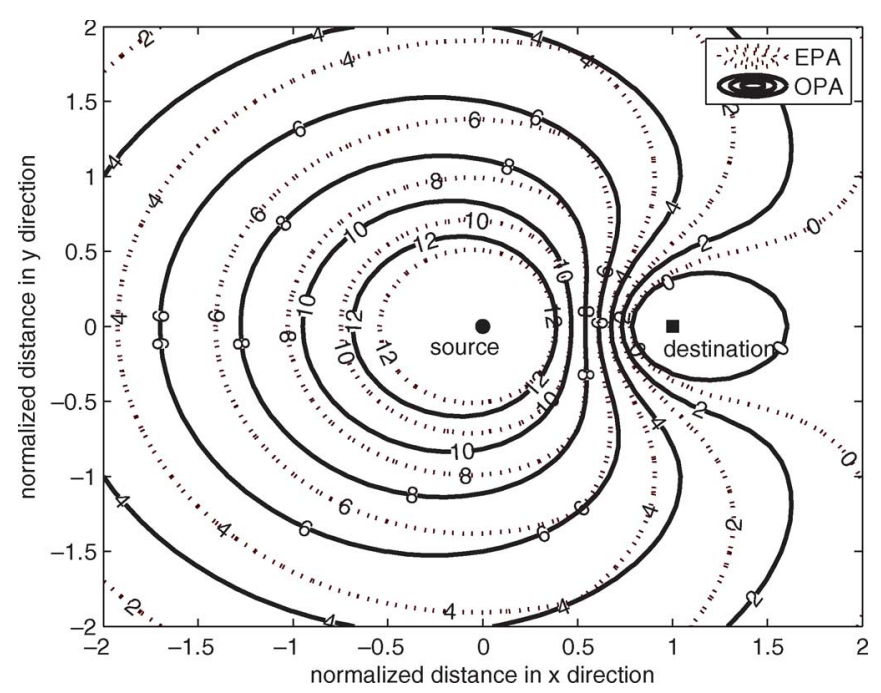

Fig. 7. Cooperative region of the regenerate and forward scheme for both power allocation.

a matching subset if there are no two edges in $\mathcal{S}$ that share the same vertex.

Our primary objective of matching is to maximize the total energy gained by cooperation in the network. The energy gain of the network is defined as the ratio of the sum of energy spent by the users when there is no cooperation to the sum of energy spent by the users with cooperation. Given the CD energy gain of any pair of users, how to maximize it in a radio cell by optimally grouping all the active users can be formulated as a nonbipartite maximum weighted (MW) matching problem, which can be solved in polynomial time $O\left(n^{3}\right)$ using the state-of-the-art MW matching algorithm [15]. Due to user mobility and intermittent traffic, the matching algorithm should periodically be executed in real time. Thus, it is important to reduce the computational and implementation complexities of the matching algorithm without compromising too much energy gain. In addition, the MW matching algorithm requires the knowledge of all interuser channel information which is 
difficult to obtain. We prefer a simpler matching algorithm which can be executed in a distributed manner.

A worst-link-first matching algorithm that maximizes the total energy gain of the pair (WLF-MaxGain) is proposed in [8]. The WLF-MaxGain algorithm gives the user, which has the worse channel condition and the higher energy consumption rate, a higher priority to choose its partner. The computational complexity of the WLF-MaxGain algorithm is $O\left(n^{2}\right)$. The WLF-MaxGain algorithm performs better than the Greedy matching algorithm in [16] for mobile networks while exhibiting a similar performance in the static networks.

For the cases of the MW and the WLF-MaxGain, the weight of a pair of users is the energy saved by cooperation between users $i$ and $j$ over no cooperation. If there is no energy saved, the two users will just use the non-cooperative scheme, and the weight of the edge linking them is zero. Thus, the weight is always non-negative, and a positive weight represents the energy gain of cooperation over no cooperation.

The question is whether using the energy gain of a pair as the weight is the best choice for the WLF matching. Consider a scenario with four users: Users $u_{1}$ and $u_{2}$ be collocated at the point $x=1$, and users $u_{3}$ and $u_{4}$ be collocated at $x=0.5$. Based on Fig. 4, with the WLF-MaxGain, $u_{1}$ and $u_{2}$ will be grouped since the energy saved by them $\left(w\left(e_{1,2}\right) \approx 440\right.$ units $)$ is the maximum among all pairs $\left(w\left(e_{1,3}\right), w\left(e_{1,4}\right), w\left(e_{2,3}\right)\right.$, $w\left(e_{2,4}\right)$, and $\left.w\left(e_{3,4}\right)\right)$, and $u_{3}$ and $u_{4}$ will be grouped thereafter. The total energy saved by the two pairs is around $440(1+$ $\left.0.5^{\alpha}\right)=495$ units. If $u_{1}$ is grouped with $u_{3}$, and $u_{2}$ is grouped with $u_{4}$, the total energy saved is around $265 \times 2=530$ units. Therefore, maximizing the energy saved by a pair does not lead to maximizing the energy saved in the network. Motivated by this example, we propose to use the maximum energy spent by the pair of users as a weight for matching, and we refer to this scheme as the WLF-MinMaxEnergy matching. The numerical results in the next section demonstrate that the performance of the WLF-MinMaxEnergy algorithm is very close to that of the optimal MW matching algorithm, and it outperforms the WLFMaxGain algorithm by a large margin.

The WLF matching algorithms are described as follows.

\section{A. Worst-Link-First by Maximizing Gain (WLF-Maxgain)}

The WLF-MaxGain matching algorithm proposed in [8] is given for easy reference.

1) The BS selects an unmatched user $i$ with the worst channel quality among all unmatched users.

2) The BS selects an unmatched user $j$ such that the energy gain $w\left(e_{i, j}\right)$ provided by the cooperation of users $i$ and $j$ over no cooperation is the maximum one among all $w\left(e_{i, k}\right)$ 's, where $k$ is an unmatched user other than $i$.

3 ) Repeat steps 1) and 2) until the number of unmatched users is less than two.

\section{B. Worst-Link-First by Minimizing Maximum Energy}

Since the WLF-MaxGain tends to maximize the energy saving by a pair of users, which is not necessarily the en- ergy saving by the user with the worst link according to the results in Section III-C, we use the maximum energy spent by the cooperating users as the weight $w\left(e_{i, k}\right)$. The matching algorithm of the WLF-MinMaxEnergy is similar to that of the WLF-MaxGain, except that the weights used for the pair of users being matched in step 2) are different: The BS selects an unmatched user $j$ such that $\max \left(E_{i}^{\mathrm{S}}+E_{i}^{\mathrm{R}}, E_{j}^{\mathrm{S}}+E_{j}^{\mathrm{R}}\right)$ is minimized among all $\max \left(E_{i}^{\mathrm{S}}+E_{i}^{\mathrm{R}}, E_{k}^{\mathrm{S}}+E_{k}^{\mathrm{R}}\right)$ 's, where $k$ is an unmatched user other than $i$. Both the WLF-MaxGain and the WLF-MinMaxEnergy algorithms have a computational complexity of $O\left(n^{2}\right)$. In step 2) of both algorithms, if it is difficult to obtain the instantaneous interuser-channel condition, the BS can use the location information and the cooperative region obtained in the previous section to choose the best partner for the user. In addition, the WLF algorithms can potentially be implemented in a distributed manner: Each user chooses its desired partner; if there is any conflict, the user far away from the BS (or has worst channel condition to the BS) has a higher priority. The unpaired users will continue to choose their desired partners from the remained unpaired users, and the procedure repeats until we cannot pair any users among the remaining unpaired ones.

It is easy to prove that the distributed WLF matching result is stable. First, the algorithm terminates with at most $\lfloor N / 2\rfloor$ iterations since each iteration will result in at least one pair. Second, for any unpaired user, it cannot break the existing pairs since at least one user of any existing pair has a higher priority than the unpaired user. Third, for any two pairs of users $\left(u_{11}, u_{12}\right)$ and $\left(u_{21}, u_{22}\right)$, they cannot exchange partners. Without loss of generality, we assume that $u_{i 1}$ has a higher priority than $u_{i 2}$, for $i=1,2$, and that $u_{11}$ has a higher priority than $u_{21}$. Since $u_{11}$ has the highest priority among all users, it can choose its desired partner $u_{12}$, and no other users can choose $u_{12}$. Thus, the matching is stable.

\section{Numerical Results}

We present the numerical results of the three matching algorithms with the OPA and the EPA for both the CD schemes in a network. We simulate a wireless network where the coordinates of the BS are $(0,0)$. The $N$ users are randomly placed on a unit disk centered at the BS, as shown in Fig. 1. Both the interuser channels (channels between two users) and the channel between a user and the BS are assumed to have a quasi-static flat Rayleigh fading. We assume that the BS can track the user locations and, thus, estimate their pairwise distances and average CSIs, which are inversely proportional to $d^{\alpha}$. By substituting $P_{\mathrm{b} 1}=P_{\mathrm{b} 2}=10^{-3}, B=128, N_{0}=1$ unit power $/ \mathrm{Hz}$, and $\alpha=3$, the average energies required for no cooperation and with cooperation are calculated. Matching is performed by the $\mathrm{BS}$ according to the $n^{2}$ weights, and the users will be grouped according to the matching results. The weights used in the MW and the WLF-MaxGain matching algorithms are the $\mathrm{CD}$ energy gains of each pair of users using the OPA strategy, and the weights used in the WLFMinMaxEnergy matching algorithm are the maximum energy levels of the pair of cooperative users. Since cooperation is not always beneficial, a pair can choose not to cooperate 


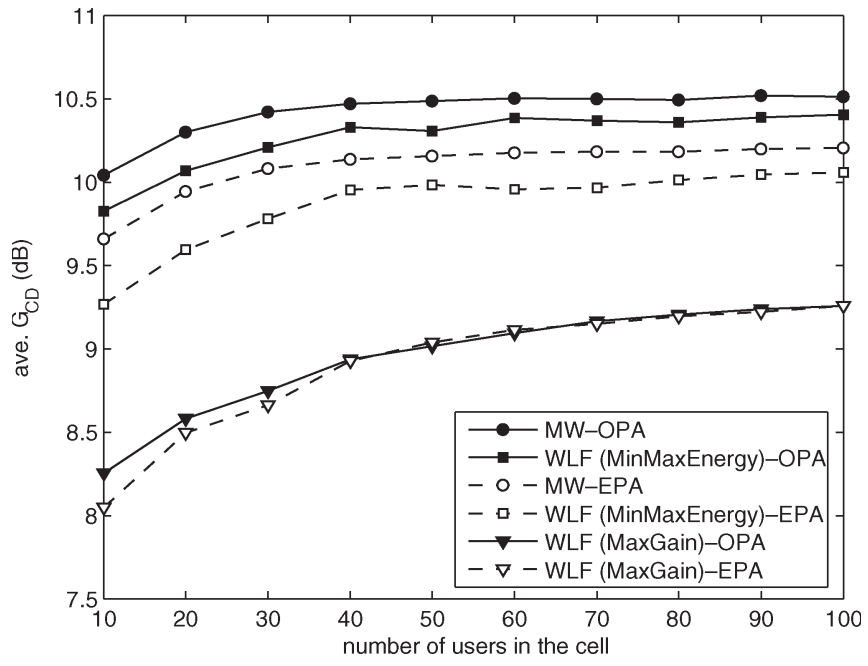

Fig. 8. Average $G_{\mathrm{CD}}$ of the amplify and forward scheme for both the OPA and the EPA.

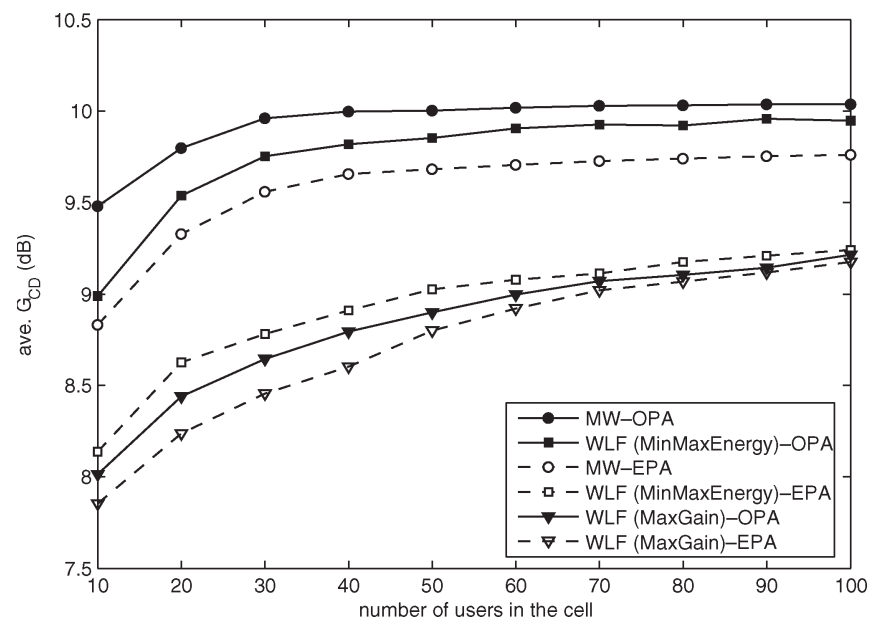

Fig. 9. Average $G_{\mathrm{CD}}$ of the regenerate and forward scheme for both the OPA and the EPA.

if there is no CD energy gain for them, and they communicate with the $\mathrm{BS}$ using the conventional noncooperative scheme.

Cell CD gain, which is the energy gain of a cell with user cooperation over a cell without user cooperation, is defined as

$$
G_{\mathrm{CD}}=10 \log _{10}\left(\frac{\sum_{i=1}^{N} E_{\mathrm{b} i}^{\mathrm{no}}}{\sum_{i=1}^{K}\left(E_{\mathrm{b} i}^{\mathrm{S}}+E_{\mathrm{b} i}^{\mathrm{R}}\right)+\sum_{i=K+1}^{N} E_{\mathrm{b} i}^{\mathrm{no}}}\right)
$$

where the first $K$ users are paired to have cooperation and the remaining $(N-K)$ users have no partners. Since $\sum_{i=1}^{N} E_{\mathrm{b} i}^{\text {no }}$ is a constant independent of matching, $G_{\mathrm{CD}}$ is maximized when $\sum_{i=1}^{K}\left(E_{\mathrm{b} i}^{\mathrm{S}}+E_{\mathrm{b} i}^{\mathrm{R}}\right)$ is minimized. Different user deployments are generated by using different random seeds. We change the number of active users in the network from 10 to 100 in order to consider both the low- and high-density scenarios. The average energy gain versus the number of users is shown in Figs. 8 and 9 for both the amplify and forward and the regenerate and forward CD schemes, respectively.

In the figures, the solid curves are those with OPA, and the dotted curves are those with EPA. Both figures show that with the MW or the WLF-MinMaxEnergy matching algorithm, the OPA can enhance the total energy gain by about 0.5 to $1 \mathrm{~dB}$ (or around 10\%-25\% improvement) over the EPA. The gap for the regenerate and forward $\mathrm{CD}$ scheme is larger than that for the amplify and forward CD scheme.

With the WLF-MaxGain matching algorithm, the difference in performance between the two power allocation schemes is negligible, particularly when the number of users in the cell is large. This is because, with the WLF-MaxGain algorithm, the BS tends to choose a partner close to the user for cooperation to maximize their cooperative energy gain. When the two users are close, their OPA result is similar to that of the EPA. On the other hand, the figures show that the performance of the WLF-MinMaxEnergy is close to that of the optimal MW matching algorithm when the OPA scheme is used. For the amplify and forward CD scheme, the performance of the WLFMinMaxEnergy with the EPA is much worse. This is because the BS tends to choose a partner sitting close to the midpoint between the user and the BS, and the results with EPA and OPA are quite different. The numerical results also demonstrate that the WLF-MaxGain performs much worse than the WLFMinMaxEnergy, as shown in Figs. 8 and 9. Furthermore, the average $G_{\mathrm{CD}}$ increases with the number of users in the low user density region and saturates in the higher user density region.

In summary, the numerical results demonstrate the importance of the combination of the power allocation and the partner selection. In order to achieve a high energy gain for the cell, the combination of OPA with the WLF-MinMaxEnergy matching algorithm is more desirable.

\section{RELATED WORK}

Most of the power allocation strategies for the CD schemes reported in the literature [1], [3]-[7] minimize error performance of the CD schemes for a fixed total power. These can be classified as fixed sum energy allocation, in which the power distribution among the source and relays is studied. These works mainly focus on the error probability reduction or coverage extension. We have proposed an optimum powerallocation strategy, which is different from the previous work, by minimizing the power consumption of the pair of users to ensure their BEP requirements (which might be different for different applications). This means that the power allocation not only finds the ratio among the partners but also minimizes the total energy consumption.

For the user grouping problem, in [12], a cooperating partner is chosen based on whether the cooperation can result in an improved frame error rate or throughput. In a multihop wireless sensor networking environment, a partner selection strategy is given in [13] based on the number of packets transmitted. The partner selection in [12] and [13] is based on optimizing the performance of a single user or a pair of users. On the other hand, centralized matching algorithms, such as minimal 
weighted, Greedy, and random, are presented for CD networks, and average outage probability given by matching is studied in [9]. In contrast, we propose a partner selection algorithm that enhances not only the individual user performance but the network performance as well. The proposed partner selection algorithm can be used in a centralized or distributed networking environment.

\section{CONCLUSION}

We have derived OPA strategies for both the regenerate and forward and the amplify and forward CD schemes, with and without the EPA constraint, and studied the optimal location of a partner. Based on the analytical results, we have also proposed a nonbipartite stable matching algorithm that can achieve a close to optimal CD gain in a wireless cellular network. We have demonstrated the effectiveness and the efficiency of the proposed WLF-MinMaxEnergy matching algorithm using the OPA strategy. It is shown that a $9 \sim 10 \mathrm{~dB} C D$ gain can be achieved, which is equivalent to prolonging the cell phonebattery recharge time by about ten times.

\section{ACKNOWLEDGMENT}

The authors would like to thank the anonymous reviewers for their valuable comments and suggestions.

\section{REFERENCES}

[1] J. Adeane, M. R. D. Rodrigues, and I. J. Wassell, "Optimum power allocation in cooperative networks," in Proc. 12th ICT, Cape Town, South Africa, May 2005.

[2] V. Mahinthan, J. W. Mark, and X. S. Shen, "Adaptive regenerate and forward cooperative diversity system based on quadrature signaling," in Proc. IEEE Globecom, San Francisco, CA, Nov./Dec. 2006, pp. 1-5.

[3] J. Luo, R. S. Blum, L. J. Cimini, L. J. Greenstein, and A. M. Haimovich, "Decode-and-forward cooperative diversity with power allocation in wireless networks," in Proc. IEEE Globecom, St. Louis, MO, Nov./Dec. 2005, pp. 3048-3052.

[4] Y. Zhao, R. Adve, and T. J. Lim, "Improving amplify-and-forward relay networks: Optimal power allocation versus selection," in Proc. IEEE ISIT, Seattle, WA, Jul. 2006, pp. 1234-1238.

[5] A. Host-Madsen and J. Zhang, "Capacity bounds and power allocation for wireless relay channels," IEEE Trans. Inf. Theory, vol. 51, no. 6, pp. 2020-2040, Jun. 2005.

[6] E. G. Larsson and Y. Cao, "Collaborative transmit diversity with adaptive radio resource and power allocation," IEEE Commun. Lett., vol. 9, no. 6, pp. 511-513, Jun. 2005.

[7] W. Su, A. K. Sadek, and K. J. R. Liu, "SER performance analysis and optimum power allocation for decode-and-forward cooperation protocol in wireless networks," in Proc. IEEE WCNC, New Orleans, LA, Mar. 2005, pp. 984-989.

[8] V. Mahinthan, L. Cai, J. W. Mark, and X. Shen, "Maximizing cooperative diversity energy gain for wireless networks," IEEE Trans. Wireless Commun., vol. 6, no. 7, pp. 2530-2539, Jul. 2007.

[9] J. N. Laneman, "Cooperative diversity in wireless networks: Algorithms and architectures," Ph.D. dissertation, Mass. Inst. Technol., Cambridge, MA, 2002.

[10] A. Scaglione, D. L. Goeckel, and J. N. Laneman, "Cooperative communications in mobile ad hoc networks," IEEE Signal Process. Mag., vol. 23, no. 5, pp. 18-29, Sep. 2006

[11] P. Herhold, E. Zimmermann, and G. Fettweis, "Cooperative multi-hop transmission in wireless networks," Comput. Netw., vol. 49, no. 3, pp. 299-324, Oct. 2005.

[12] Z. Lin, E. Erkip, and A. Stefanov, "Cooperative regions and partner choice in coded cooperative systems," IEEE Trans. Commun., vol. 54, no. 7, pp. 1323-1334, Jul. 2006
[13] N. Shastry, J. Bhatia, and R. S. Adve, "Theoretical analysis of cooperative diversity in wireless sensor networks," in Proc. IEEE Globecom, St. Louis, MO, Nov./Dec. 2005, vol. 6, pp. 3269-3273.

[14] R. K. Ahuja, T. L. Magnanti, and J. B. Orlin, Network Flows: Theory, Algorithms, and Applications. Englewood Cliffs, NJ: Prentice-Hall, 1993.

[15] H. N. Gabow, "An efficient implementation of Edmonds' algorithm for maximum matching on graphs," J. ACM, vol. 23 , no. 2, pp. 221-234, Apr. 1976.

[16] D. Avis, "A survey of heuristics for the weighted matching problem," Networks, vol. 13, no. 4, pp. 475-493, 1983.

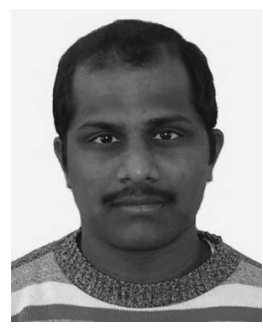

Veluppillai Mahinthan (S'02-M'08) was born in Jaffna, Sri Lanka. He received the B.Sc.Eng. degree in electrical and electronic engineering from the University of Peradeniya, Peradeniya, Sri Lanka, in 2000 and the M.Eng. degree from the National University of Singapore (NUS), Singapore, in 2003, and the $\mathrm{Ph} . \mathrm{D}$. degree from the University of Waterloo (UW), Waterloo, ON, Canada, in 2007, both in electrical and computer engineering.

From 2000 to June 2001, he was an Instructor with the Department of Electrical and Electronic Engineering, University of Peradeniya. From July 2001 to August 2003, he was a Research Scholar with the Institute for Infocom Research $\left(\mathrm{I}^{2} \mathrm{R}\right)$, Singapore. From September 2003 to September 2007, he was a Research Assistant with the Centre for Wireless Communication, UW. Since October 2007, he has been a Postdoctoral Fellow with the Department of Electrical and Computer Engineering at UW. His current research interests involve wireless communications, including cooperative diversity, space-time coding, implementation aspects and performance analysis of cross-layer communications, and channel estimation and signal processing for communications.

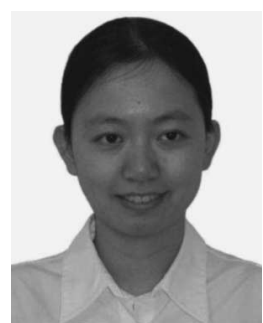

Lin Cai (S'00-M'05) received the M.A.Sc. and $\mathrm{Ph} . \mathrm{D}$. degrees (with outstanding achievement in Graduate Studies Award) in electrical and computer engineering from the University of Waterloo, Waterloo, ON, Canada, in 2002 and 2005, respectively.

Since July 2005, she has been an Assistant Professor with the Department of Electrical and Computer Engineering, University of Victoria, Victoria, BC, Canada. Her research interests span several areas in wireless communications and networking, with a focus on network protocol and architecture design supporting emerging multimedia traffic over wireless, mobile, ad hoc, and sensor networks.

Dr. Cai is a member of the Association for Computing Machinery. She serves as an Associate Editor for the IEEE TRANSACTIONS ON VEHICULAR TECHNOLOGY, the EURASIP Journal on Wireless Communications and Networking, and the International Journal of Sensor Networks. 


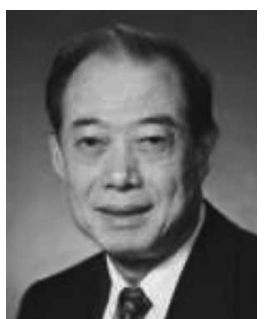

Jon W. Mark (S'60-M'62-SM'80-F'88-LF'03) received the B.A.Sc. degree in electrical engineering from the University of Toronto, Toronto, ON, Canada, in 1962 and the M.Eng. and Ph.D. degrees in electrical engineering from McMaster University, Hamilton, ON, in 1968 and 1970, respectively.

From 1962 to 1970, he was an Engineer and then a Senior Engineer with the Canadian Westinghouse Company Ltd., Hamilton, ON. Since September 1970, he has been with the Department of Electrical and Computer Engineering, University of Waterloo, Waterloo, ON, where he is currently a Distinguished Professor Emeritus. He served as the Department Chairman from July 1984 to June 1990. In 1996, he established the Centre for Wireless Communications at the University of Waterloo and is currently serving as its Founding Director. He had been on sabbatical leaves at the following places: IBM Thomas J. Watson Research Center, Yorktown Heights, NY, as a Visiting Research Scientist from 1976 to 1977; AT\&T Bell Laboratories, Murray Hill, NJ, as a Resident Consultant from 1982 to 1983; Laboratoire MASI, Université Pierre et Marie Curie, Paris, France, as an Invited Professor from 1990 to 1991; and the Department of Electrical Engineering, National University of Singapore, Singapore, as a Visiting Professor from 1994 to 1995 . He has previously worked in the areas of adaptive equalization, image and video coding, spread-spectrum communications, computer communication networks, ATM switch design, and traffic management. He recently coauthored the book Wireless Communications and Networking (Prentice-Hall, 2003). His current research interests are in broadband wireless communications, resource and mobility management, and cross-domain interworking.

Dr. Mark was an Editor of the IEEE TRANSACTIONS ON COMMUNiCATIONS from 1983 to 1990, a member of the Inter-Society Steering Committee of the IEEE/ACM TRANSACTIONS ON NETWORKING from 1992 to 2003, a member of the IEEE Communications Society Awards Committee from 1995 to 1998, an Editor of Wireless Networks from 1993 to 2004, and an Associate Editor of Telecommunication Systems from 1994 to 2004. He was the recipient of the 2000 Canadian Award for Telecommunications Research and the 2000 Award of Merit of the Education Foundation of the Federation of Chinese Canadian Professionals.

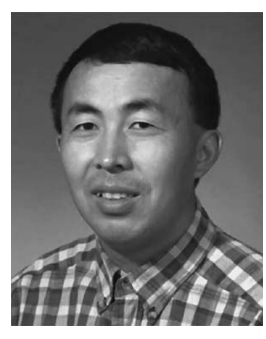

Xuemin (Sherman) Shen (M'97-SM'02) received the B.Sc. degree in electrical engineering from Dalian Maritime University, Dalian, China, in 1982, and the M.Sc. and Ph.D. degrees in electrical engineering from Rutgers University, Camden, NJ, in 1987 and 1990, respectively.

$\mathrm{He}$ is a Professor and the Associate Chair for Graduate Studies, Centre for Wireless Communications, Department of Electrical and Computer Engineering, University of Waterloo, Waterloo, ON, Canada. His research focuses on mobility and resource management in interconnected wireless/wired networks, ultrawideband wireless communication systems, wireless security, and ad hoc and sensor networks. He is a coauthor of three books and has published more than 300 papers and book chapters in wireless communications and networks, control, and filtering.

Dr. Shen serves as the Technical Program Committee Chair for IEEE Globecom'07, the General Cochair for Chinacom'07 and QShine'06, and the Founding Chair for the IEEE Communications Society Technical Committee on P2P Communications and Networking. He also serves as a Founding Area Editor of the IEEE TRANSACTIONS ON WIRELESS COMMUNICATIONS; the Editor-in-Chief of the Peer-to-Peer Networking and Application; an Associate Editor of the IEEE TRANSACTIONS ON VeHICULAR TECHNOLOGY, the KICS/IEEE JOURNAL OF COMMUNICATIONS AND NETWORKS, COMPUTER NETworks, ACM/Wireless Networks, Wireless Communications and Mobile Computing (Wiley), etc. He has also served as a Guest Editor for the IEEE Journal on SELECTEd AREAS IN COMMUNiCATIONS, IEEE Wireless COMmUnications, and the IEEE Communications Magazine. He was the recipient of the Excellent Graduate Supervision Award in 2006 and the Outstanding Performance Award in 2004 from the University of Waterloo, the Premier's Research Excellence Award in 2003 from the Province of Ontario, and the Distinguished Performance Award in 2002 from the Faculty of Engineering, University of Waterloo. He is a Registered Professional Engineer in Ontario. 